VIROLOGY

\section{Re-emergence of canine distemper in wildlife in Belgium}

BETWEEN 30 and 50 red foxes (Vulpes vulpes) are submitted each year to the University of Liège's veterinary faculty for postmortem examination. Occasionally, other wild carnivores such as European badgers (Meles meles) and raccoons (Procyon lotor) are also submitted.

A PCR-based screening for canine distemper virus $(C D V)^{1}$ has been performed systematically. Since 2014 , all submitted cases tested negative for CDV. On November 14, 2017, a wild raccoon with severe neurological impairment was euthanased in a revalidation centre, near the Liège veterinary faculty.

The histopathological evaluation of the brain revealed a moderate non- suppurative meningoencephalitis with severe demyelination of white matter tracts (Fig 1). The other organs were not remarkable. Brain samples were positive for the presence of CDV RNA by real time PCR. ${ }^{1}$

From November 2017 to March 2018, a total of 13 red foxes were submitted for postmortem examination, among which six had a history of abnormal behaviour or collapse and so were euthanased for ethical reasons. Typical neuropathological lesions were present in brain tissues of 10 of these cases, all of which tested positive by real time PCR for CDV RNA.

All but one of the CDV-positive foxes originated from within a $15 \mathrm{~km}$ radius around Liège. The other three foxes were PCR-negative, two of them coming from outside the affected area.

To exclude the possible circulation of a vaccine strain, a conventional RT-PCR was used, ${ }^{2}$ with Sanger sequencing of the 294-bp amplicons. A BlastN analysis (https://blast.ncbi. $\mathrm{nlm}$.nih.gov/Blast.cgi) revealed that all cases belonged to a single strain, sharing 96 per cent of nucleotide identity to the closest strain available in GenBank (accession number AY386315.1). This finding did not support the hypothesis of the circulation of a vaccine strain.

The lack of detection of CDV in all wild carnivores tested over the past few years until these cases in southern Belgium suggests a low prevalence of this virus. The current outbreak, concentrated over a small geographical area, with a high mortality in red foxes, is highly suggestive of a re-emergence of CDV in a naive population.

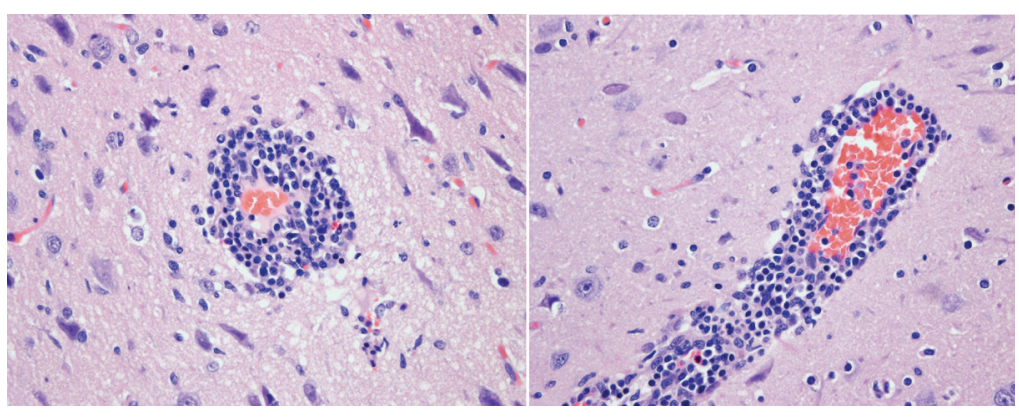

Fig 1: Histopathology of the brain of a canine distemper virus-infected raccoon from Liège, showing non-suppurative meningoencephalitis with prominent perivascular cuffing. Haematoxylin and eosin, $x 400$

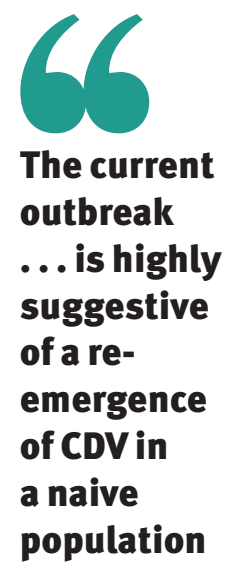

New cases are expected in the coming weeks and a surveillance programme in neighbouring areas has been initiated. Further phylogenetic analyses will be implemented to determine the origin of the strain detected in these 11 (10 fox, one raccoon) cases. Finally, such a re-emergence provides further arguments to pursue the promotion of vaccination of domestic dogs against CDV.

Mutien Garigliany, Michaël Sarlet, Mathieu Franssen, Daniel Desmecht, Department of Pathology,

Rosario Volpe, Christophe Lesenfants, Julien Paternostre, Annick Linden, Surveillance Network for Wildlife Diseases, Faculty of Veterinary Medicine, University of Liège, Liège, Belgium

e-mail: a.linden@uliege.be

\section{References}

1 ELIA G, DECARO N, MARTELLA V, et al. Detection of canine distemper virus in dogs by real-time RT-PCR. J Virol Methods 2006;136:171-6

2 WILKES RP, SANCHEZ E, RILEY MC, KENNEDY MA. Real-time reverse transcription polymerase chain reaction method for detection of canine distemper virus modified live vaccine shedding for differentiation from infection with wild-type strains. / Vet Diagn Invest 2014;26:27-34

doi: 10.1136/vr.k1610 